\title{
Characterization of intensity and spatial variations along coronal loops
}

\section{A TRACE case study}

\author{
A. Borgazzi ${ }^{1}$ and A. Costa ${ }^{1}$ \\ Instituto de Astronomía y Física del Espacio, CONICET, Argentina \\ e-mail: costa@iafe.uba.ar \\ Received 1 November 2004 / Accepted 30 May 2005

\begin{abstract}
We describe dynamical features and evolutionary characteristics of brightening coronal loops. We describe intensity variations, both in space and time, along a coarse grain loop structure, confirming high speed velocity scenarios. We apply the method to TRACE space-born images that show a compound of several magnetic threads. MICA ground-based images display a unique non-resolved loop structure. We confirm that a coherent behavior of the intensity along neighboring magnetic tubes occurs, i.e. we obtain a similar pattern from both telescopes: each has two branches, suggesting the sliding down of plasma in both directions from a given position on the loop structure. The apparent sliding down occurs in approximately $12 \mathrm{~min}$. After the first appearance, TRACE registers two reiterations of the phenomenon suggesting a wave-based explanation. The feasibility of wave-based and flow-based models is analyzed. In either case, in order to explain the coherent coronal behavior the scenario of apparently non-interacting coronal threads requires theoretical explanations that consider uniform chromospheric conditions covering the footpoints of all the related magnetic tubes. We suggest a characteristic longitude of coherence.
\end{abstract}

Key words. Sun: corona - waves - Sun: magnetic fields - instabilities

\section{Introduction}

The theoretical modeling and the interpretation of observations of coronal loop systems deal, among others, with three main discussions: 1) whether the propagating disturbances in loops and post-flare loops are waves or plasma flow; 2) whether the disturbances are triggered from the top or the bottom of the loop systems; and 3) what is the resulting appearance of a multi-loop complex system.

On the one hand, dynamical features of brightening coronal loops have been traditionally interpreted as field-aligned flow of matter generated by asymmetries in the energy input. Among the different interpretations, most classical theoretical models have difficulties in determining the physical conditions that make them compatible with observations. Both static loops and steady state models - for the two classes of temperatures models: hot (isothermal coronas with $T \approx 10^{6} \mathrm{~K}$ ) and cool (gradually increasing temperatures up to $T \approx 10^{5} \mathrm{~K}$ ) - fail to provide a satisfactory explanation of both the emission measure distribution and the Doppler shift observations (Jordan 1980; Serio 1981; Craig \& McClymont 1978; Mariska 1984). Thus, this suggests that in traditional model scenarios radiative losses cannot be compensated by thermal conduction. Therefore, other heating mechanisms must be assumed (Aschwanden et al. 1999, 2000; Walsh \& Galtier 2000).
Also, theoretical time-dependent models of individual loops where the plasma evolves in response to a cyclical process of heating and cooling of the flow have difficulties in fitting observations (Klimchuk \& Mariska 1988).

On the other hand, the assumption of propagating disturbances associated with slow magneto-acoustic waves in high Alfvén speed media is a relatively recent field of investigation. Several wave-based models were developed to explain observations (Nakariakov et al. 2000; Tsiklauri \& Nakariakov 2001). These authors suggest that upwardly propagating waves (of observed periods between 5-20 min) that decay significantly in the vicinity of the loop apex explain the rarity of observational detection of downwardly propagating waves. However, upwardly propagating disturbances with non-decaying or even growing amplitudes were observed in coronal EIT plumes. Analytic models have shown that slow magneto-acoustic waves may be trapped and nonlinearly steepened with height, providing a possible interpretation of the phenomenon (Ofman et al. 1999).

However, due to the intensity of the flaring, the plasma dynamic of flare loops is generally associated with flows rather than with waves. In fact, systematic intensity perturbations in post-flare loops can suggest that they are the result of evaporation-condensation cycles caused by the efficient heating of the flaring plasma from the chromosphere. 
Thus, chromospheric evaporation seems to be the main initial matter inflow source for flare loops. De Groof et al. (2004) analyzed an off-limb half loop structure from an EIT shutterless campaign and gave arguments to rejected the slow magnetoacoustic description and to support the flowing/falling plasma one. Nevertheless, these authors admit that the wave theory cannot be excluded yet.

Other authors have suggested that a combination of phenomena can be at the basis of a better interpretation. Alexander et al. (1998) examined 10 flares and concluded that plasma turbulence could be the source of the observed intensity changes rather than hydrodynamic flows such as chromospheric evaporation. They pointed out that it cannot be excluded that there is a degree of "gentle evaporation" occurring early in the event with associated hard X-ray emission below their threshold of detection. A series of more recent papers (Tsiklauri et al. $2004 a, b, c)$ that combine theoretical and observational analysis showed that oscillations in white, radio and X-ray light curves observed during solar and stellar flares may be produced by slow standing magneto-acoustic modes of the loops. They found that a transient heat deposition at the loop bottom - or at the apex - leads to a posterior up-flow evaporation of material of the order of a few hundreds of $\mathrm{km} \mathrm{s}^{-1}$. During the peak of the flare, the simulations showed that a combined action of heat input and conductive and radiate losses could yield an oscillatory pattern with typical amplitudes of up to a few tens of $\mathrm{km} \mathrm{s}^{-1}$. Then, a cooling phase of plasma draining with velocities of the order of hundreds of $\mathrm{km} \mathrm{s}^{-1}$ occurrs. The numerical quasiperiodic oscillations in all the physical quantities, that resemble observational features, were interpreted as being produced by standing sound waves caused by impulsive and localized heating.

A related topic of interest is the appearance of multi-loop complex systems. Due to the fact that individual loops in loop systems are thought of as having their own hydrodynamic evolution and being thermodynamically isolated, with independent timing and physical parameters, the triggering mechanism that gives rise to the coherent behavior of most observations is unclear. Kjeldseth-Moe \& Brekke (1998) proposed a dynamic scenario where observed individual active region loops are considered composed of strands of finer structures or magnetic tubes, each one with a resolution that is below the resolution of SOHO instruments. The thin magnetic tubes could have gas flowing in different directions with different temperatures and with different speeds. This scenario suggests coarse-grained, unresolved fine-structured tubes, mostly magnetically isolated from each other and of a global quasi-isothermal appearance that could theoretically justify the large velocities observed. Moreover, Schrijver (2001) has described frequent catastrophic cooling and evacuation of quiescent solar coronal loops in active regions (occurring almost once every 2 days), which seems to be triggered when the loop heating is reduced by 1.5 orders of magnitude. Relatively cool material seems to move downwards at speeds of up to $100 \mathrm{~km} \mathrm{~s}^{-1}$. He also suggested that this scenario is compatible with the existence of several thin cool strands embedded within an initial loop. However, he established that the filamentary structure must behave in a coherent manner. Hara et al. (2004) found that the appearance of transition region loops (up to $1 \mathrm{MK}$ ) are related to small-scale flaring in the corona resembling the cooling of these loops by material draining from the loop top.

In a previous paper (Paper I: Costa \& Stenborg 2004), we presented a method, and corresponding results, that allows to measure intensity variations along the structure of localized small scale coronal (post-flaring) loops observed with the MICA telescope (Mirror Coronagraph for Argentina, Stenborg 1999 ) in the well known green coronal line at $530.3 \mathrm{~nm}$. The technique allowed the tracking of the plasma along the loop under study, provided the shift of the excess intensity detected on it is a trace of the movement of the excess density. Otherwise, it allowed the determination of the speed at which the plasma inside the loop changes its temperature to make it visible at the wavelength of observation. It proved to be a reliable technique to determine speeds of the intensity variations detected along a given magnetic structure.

The method was applied to a loop system on the south-west limb on green line images taken on October 1st, 2001 with a cadence of about one per minute. Part of the structure showed a brightening near the top, which moved down along its axis with mean velocities that suggest scenarios of high-speed plasma flows (Wood et al. 1998; Kjeldseth-Moe \& Brekke 1998; Schrijver 2001). The mean velocity values obtained were $41 \mathrm{~km} \mathrm{~s}^{-1}$ for one branch and $140 \mathrm{~km} \mathrm{~s}^{-1}$ for the other one indicating the descent of the flow or the intensity variation in both directions. Thus, we could characterize the movement of the brightening along the loop as a function of time.

The aim of Paper I was to derive a reliable procedure to analyze potential intensity variations along coronal loops that could be applied to any sequence of images obtained with instruments capable of providing images of loop-like structures with high temporal resolution. In this work we apply the Paper I method to images obtained with the Transition Region and Coronal Explorer, TRACE telescope, (Handy et al. 1999). Thus, the intentions of this work are, to corroborate Paper I results, to provide a more accurate description of multi-loop systems by comparison of simultaneous observations obtained with space and land telescopes and to reexamine the flow hypothesis for post-flaring loops. We also want to acquire insight into the general physical information that can be obtained from the method. We chose the case studied in Paper I i.e., the October 1st, 2001 event, so as to be able to compare information about almost the same physical system and to test the predictive power of the method via its application to other instruments.

\section{Observations}

The October 1st, 2001 event started around 04:40 UT in the vicinity of the NOAA AR 9628 (22 S, $91 \mathrm{~W})$ and AR 9632 $(21 \mathrm{~S}, 73 \mathrm{~W})$. An M9.1 flare that peaked in both the low and high energy bands between 05:13 UT and 05:16 UT was reported by the GOES 10 satellite. The recovery phase lasted until the end of the day and corresponding active regions, associated with Fe XII EIT/SOHO images (Delaboudiniere et al. 1995), were seeing flaring all day. 
MICA observation started at 11:48 UT were not able to record the early development of the event though the recovery phase events were well observed. The MICA green line images considered for the analysis were those where, by visual inspection, the post-flare loops seemed to be stabilized. That was observed to occur in the images taken from 17:19:14 UT to 19:27:00 UT.

MICA and TRACE telescopes have different space and time resolution and the phenomenon was observed in different coronal lines (MICA line Fe XIV: $\lambda_{\mathrm{M}}=530 \mathrm{~nm}, T_{\mathrm{M}}=1.8 \times$ $10^{6} \mathrm{~K}$ and TRACE line Fe IX: $\lambda_{\mathrm{T}}=17.1 \mathrm{~nm}, T_{\mathrm{T}}=1 \times 10^{6} \mathrm{~K}$ ). The TRACE image resolution is approximately 0.5 arcsec and the imaging cadence of about $1 \mathrm{~min}$. Unfortunately, as TRACE images suffered from radiation noise spikes, the time interval that could be used had a lack of information of $10 \mathrm{~min}$ with respect to MICA's. The images were normalized and treated with a median filter in order to avoid white spots produced by the incidence of high energetic particles on the CCD camera. The smoothing replaces each point with the median of the two-dimensional neighborhood pixels of a given width. However, MICA's time resolution was better than the TRACE one due to the fact that, for the purposes of the method, a number of these images were corrupted by high energy particles (i.e., there is a lack of images for the time intervals from 17:57:00 UT to 18:07:10 UT and 17:41:19 UT to $17: 43: 22 \mathrm{UT})$.

With the MICA telescope (Paper I), from all of the features present in the region under study, we distinguished three different structures (see Fig. 1a) i) the loop marked I in Fig. 1a; ii) the interacting pattern marked as II in the same figure; iii) the pattern marked as III that corresponds to the top of a set of postflare loops not resolved by MICA. We will discuss the white features or cuts of Structure I in Fig. 1a in the next section.

A general qualitative correlation can be established between the images obtained with both telescopes (MICA image of the 18:00 UT and TRACE image of the 18:07 UT) as can be seen in Fig. 1. The correlated features are indicated with the same numbers in Figs. 1a and 1b. What seems a unique loop (I) in the MICA image is a cluster of magnetic tubes in the TRACE one. In this paper we analyze the feature labeled I in both figures. We also analyze Structure IV for TRACE images. For a description of the MICA telescope and for information about image treatment and the stabilization procedure see Paper I.

To confirm the correspondence of the features shown in Fig. 1 and to establish a correspondence between pixels of the images, we took a contour profile of the TRACE image at 18:07 UT and performed a co-alignment with the MICA image at 18:00 UT ( $\pm 1-2$ MICA pixels). The result is displayed in Fig. 2. The straight line on the figure indicates the apex position of Structure I, as described by the two telescopes.

\section{Method}

In this section we describe the method and its application to MICA images. The application to TRACE images is straightforward. From the whole time sequence recorded by MICA we selected a sub-interval (from 17:19 UT to 19:27 UT) in which

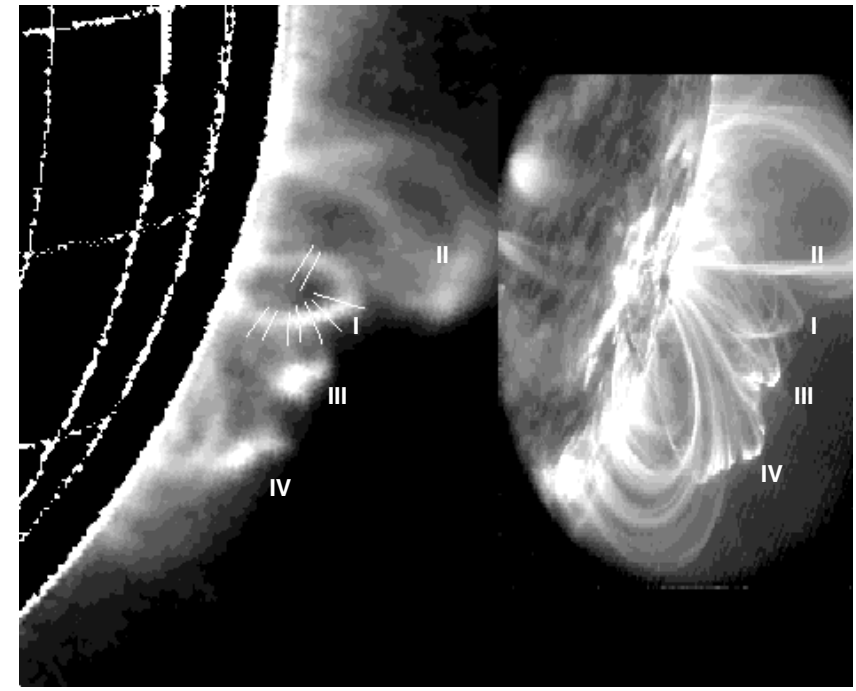

Fig. 1. a) MICA $530.3 \mathrm{~nm}$ green line image (18:00 UT) showing the structures I-IV. The selected cuts were numbered clockwise beginning from the first one of the Northern loop's leg. b) TRACE $17.1 \mathrm{~nm}$ image (18:07 UT) showing the Structures I-IV.

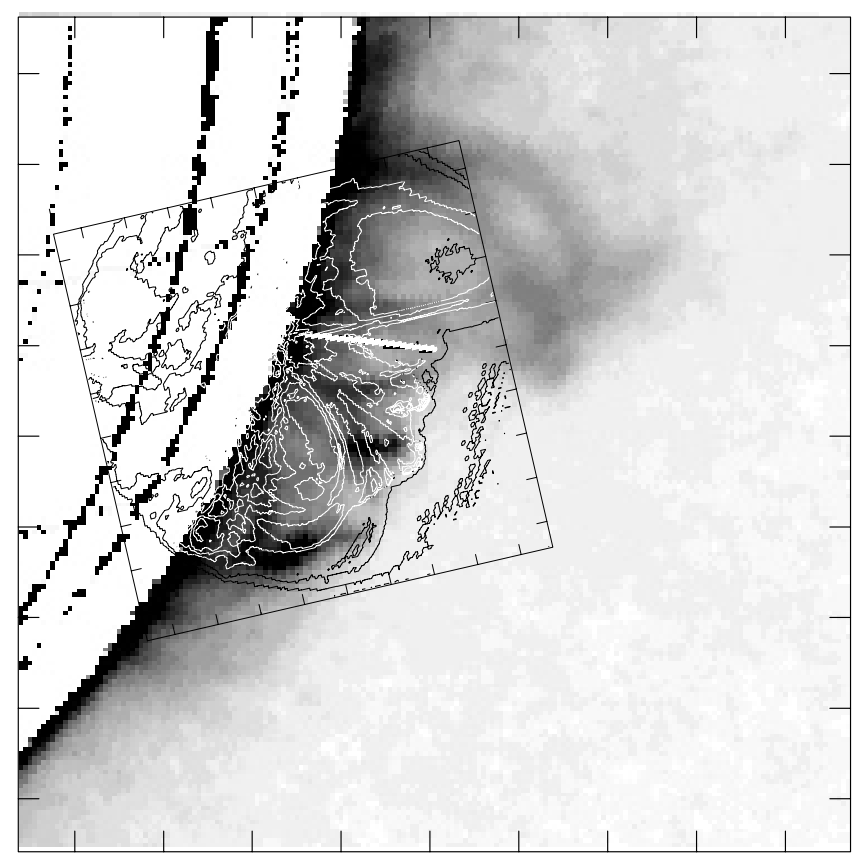

Fig. 2. Superposition of the TRACE 18:07 UT image and the MICA 18:00 image. The straight line indicates the location of the apex of Structure I in both figures. Each division of MICA's image axis corresponds to $20 \mathrm{Mm}$; each division of TRACE's image axis corresponds to $10 \mathrm{Mm}$.

the magnetic feature of Structure I was stationary in time (see Paper I). However, intensity variations along the green line loop could be detected. Therefore, we characterized the temporal evolution of this intensity variation along the loop.

The intensity profiles of the cross section of the loop at nine different positions on the first green line image of the sequence (i.e., at 17:19 UT) were obtained. The white straight lines shown on the loop-like structure marked as I in Fig. 1a depict the positions where the cuts (hereafter $M_{j}, j=1 \ldots 9$ ) were 
made. The same procedure was performed on the TRACE images when different threads intersected a cut (hereafter $T_{j}$, $j \approx 1 \ldots 20$ ). Each MICA cut $M_{j}$ was considered acceptable if the intensity profile during it exhibited only one relative maximum along the whole temporal sequence. In MICA images this procedure guaranteed that the maximum of the intensity profile indicated the center of the loop, provided that only the loop under consideration is crossed by the cut (see Fig. 3a). TRACE images have a better space resolution than MICA, thus, one MICA cut generally covers several non resolved threads which are tenuously resolved by TRACE. This results in differences between the regularities of the two profiles obtained when the intensity along the cut is displayed (Figs. 3a and 3b). To avoid large macroscopic and coherent space displacements of the magnetic structure we imposed on MICA images the condition that the intensity along the cut must exhibit only one relative maximum during the temporal sequence. For TRACE images we imposed the condition that the intensity along each cut of the temporal sequence must exhibit a quadratic-like shape with a maximum at the center. Otherwise, if the shape of the wings changed such that the quadratic-like form was relaxed, suggesting a space displacement of the magnetic structure, the series was time reduced in order to guarantee stability. This procedure was implemented (Paper I) to determine the MICA time interval were the magnetic structure remained stable i.e. from 17:19:14 UT to 19:27:00 UT. Moreover, a test to discriminate the movement of the plasma from the movement of the magnetic structure as a whole was developed in Paper I. When we examined the TRACE images in the same temporal interval as MICA's we found that all the cuts fulfill the TRACE requirement.

Once the spatial position of the cuts are chosen based on the first image (considered to be the start of the sequence), the intensity profile of the cross section of the loop at the coordinates covered by each cut is computed for each image in the sequence. The spatial coordinates, $x(j, t)$ and $y(j, t),(j$ and $t$ being the number of the cut and the time of the image, respectively) that correspond to the place on each image where the intensity maximum $I_{\mathrm{m}}(j, t)$ occurs, identify the center of the flux tube throughout the temporal sequence.

When following the temporal behavior of $I_{\mathrm{m}}(j, t)$ along the whole image sequence - each image corresponding to a different time - for each cut there is a maximum value. This particular feature represents the brightening we are investigating. To clearly identify the time of such a maximum value a second order polynomial is fitted to the points surrounding the corresponding absolute maximum (five points on either side of the absolute maximum were chosen). The position of the maximum of the resulting parabola $\left(t_{j}\right)$ is considered to be the time position of the feature that characterizes the brightening. In MICA images the time where such a maximum occurred was slightly different for each cut, indicating that the brightening moved along the loop.

Figure 5a shows, for MICA images, that at the time $t_{0}=$ 18:00 UT the loop had its maximum brightness at $d_{0}=56 \mathrm{Mm}$. This distance was measured considering a virtual axis along the loop; the origin of the axis was taken to be the position of the first cut on the northern leg of Fig. 1a. For $t_{j}>t_{0}$ the
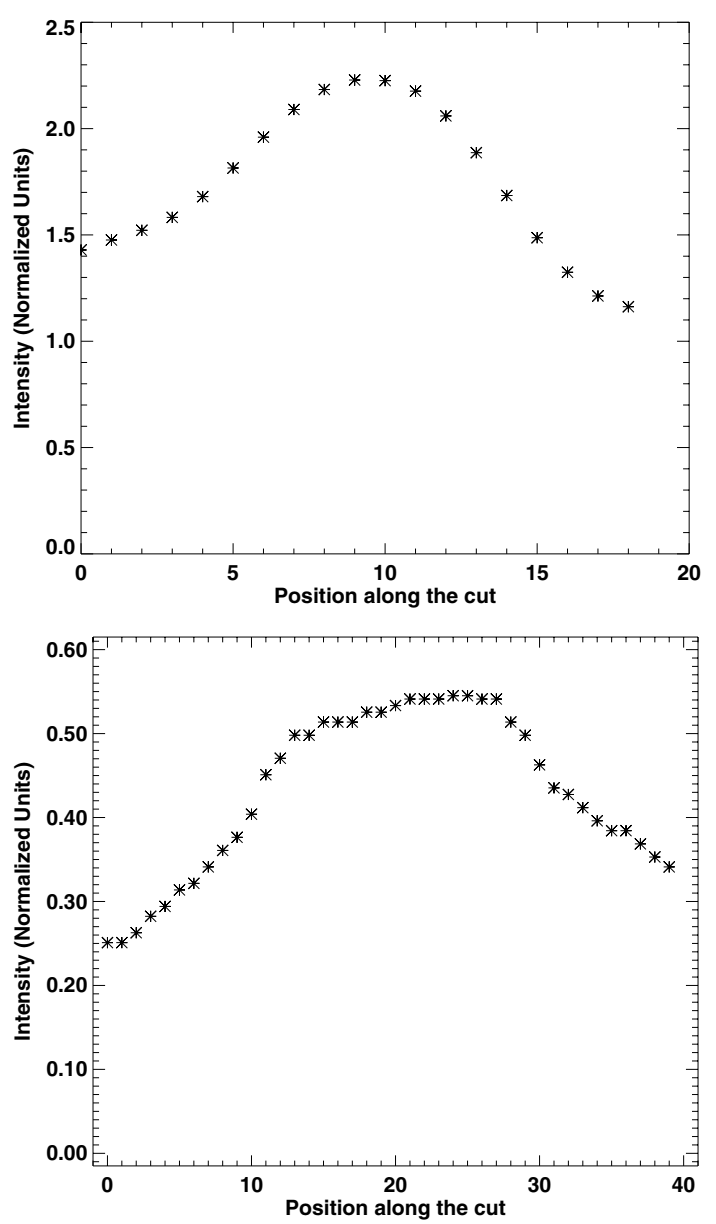

Fig. 3. a) Typical MICA intensity profile as a function of the number of pixels covered by a cut. b) The same for TRACE images.

maximum occurred at two different positions, one on each side of the point located at $d_{0}$. Likewise, the trend of two maxima continued with time. Note that, even when the whole MICA time series spanned from 17:19:14 UT to 19:27:00 UT the procedure results in a shorter interval. Figure 5a shows the time interval where each cut in the series had its maximum showing that Structure I had its greatest activity during this interval.

\section{Results}

In this section we show the results of applying the procedure described in last section to the sequence of TRACE images. We made 20 cuts covering Structure I of Fig. 1b. We then followed the procedure summarized in last section and extensively described in Paper I.

When we plotted the intensity of each cut as a function of time we found a notable difference with MICA results. In Fig. 4a there are two types of characteristic features or intensity perturbations present in all the cuts. While in the MICA case (Fig. 4b) we obtained a unique intensity maximum for each cut over the time interval, in the TRACE case we note two main significant sub-intervals where a maximum occurs. Thus, we divided the whole interval in two and we applied the method to each of the sub-intervals. Note that this is done because 

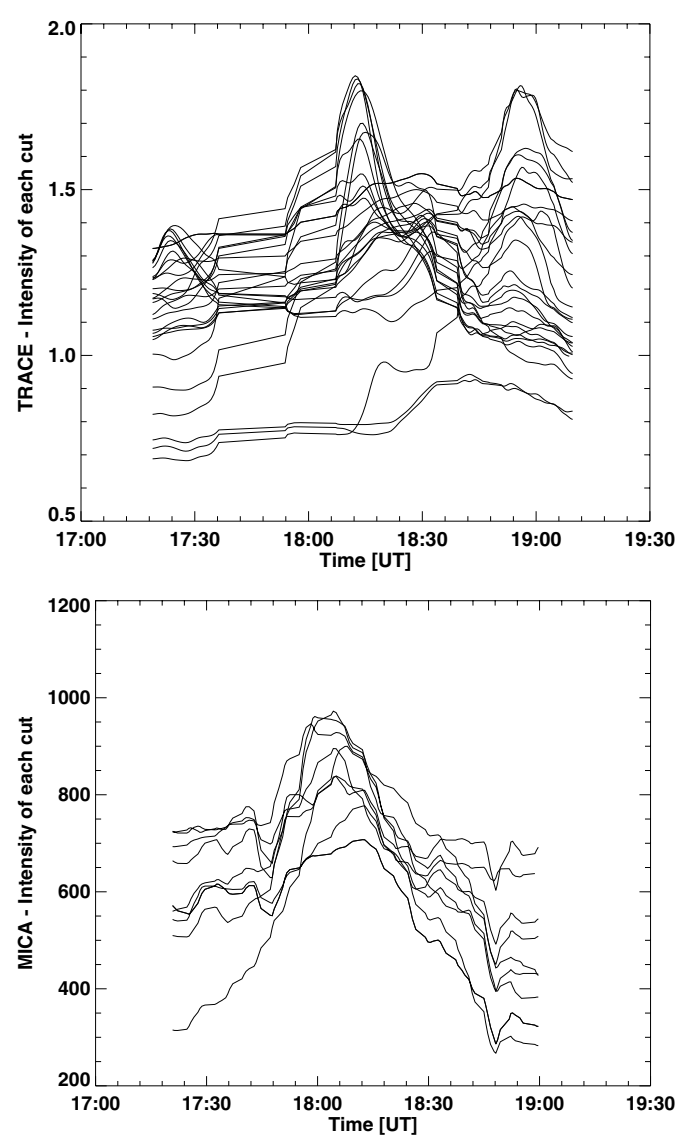

Fig. 4. a) TRACE intensity of each cut as a function of time. b) MICA intensity of each cut as a function of time.

the method registers the maxima of the cuts and thus, if the intention is to analyze features that are not the most intense ones, then the less intense intervals must be isolated so that relative maxima become absolute maxima.

Figure $5 \mathrm{~b}$ shows the location of the brightening as a function of time for the sub-interval ranging from $\approx 18: 12 \mathrm{UT}$ to $18: 27 \mathrm{UT}$ and measured along a virtual axis that extends along the whole complex loop composed of several thin magnetic tubes covered by the cuts. As was mentioned, the origin of the axis is taken to be the location of the first cut and the distance is measure along the loop (see Paper I). Thus, Fig. 5b shows the time sub-interval where the major TRACE activity of Structure I occurs. The fact that more than one brightening occurs at approximately the same hour in Fig. 5b indicates that more than one cut at the same time has its maximum. Thus, more than one neighboring coronal isolated magnetic tube has its maximum brightening at that hour. The time correlation of the brightening of neighboring threads reflects a coherent behavior between them. This coherence i.e. the mean decrease of the brightening in each branch, is in accordance with the two branch patterns obtained with MICA. Also, in Fig. 5a, the occurrence of a brightening in cuts 2 and 3 (the order of the cuts is that of the ordinates) at the same hour could indicate that at least two non-resolved MICA threads, covered by different cuts, had their maximum at the same hour.

While we obtained a similar behaviour with TRACE as with MICA (Fig. 5a) in the TRACE case the pattern was time
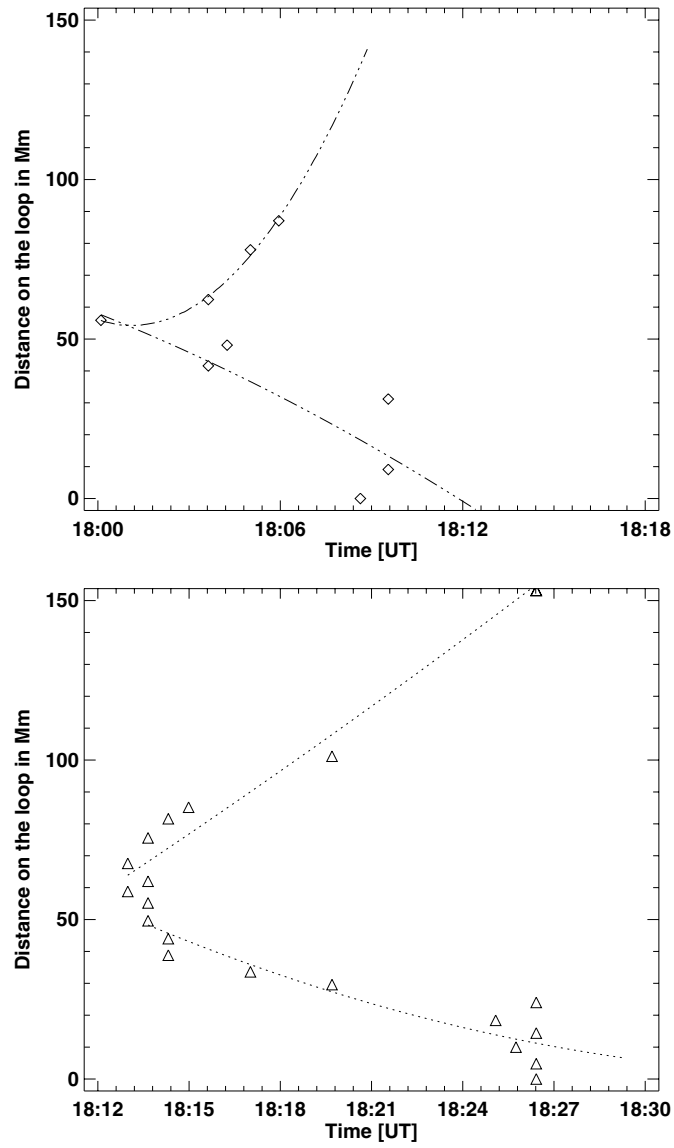

Fig. 5. MICA location of the brightening, measured along the loop, as a function of time. The spatial origin is chosen to be the position of the first cut traced over the loop's Northern leg. The time origin matches the occurrence of the first maximum, which is cut number 6 . b) The same for TRACE. The first maximum was cut number 13 . The upper curves in both figures corresponds to the southern branch and the lower ones to the northern branch of Fig. 1 i.e., each of the plasma flow directions along the loop. In the figure the apex of the Structure is located at $31 \mathrm{Mm}$ (it was measured along the loop from the first co-aligned cut of both type of images).

displaced from MICA's one. Thus, in order to be sure we were comparing the same event, we isolated the TRACE images that corresponded to the MICA time interval where the maxima occurred (see Fig. 5a). The TRACE signal corresponding to this interval was weaker and is poorly distinguished in Fig. 4a. An additional inconvenience for the comparison is that there is a gap of $10 \mathrm{~min}$ between the two telescopes.

Thus, we applied the method to each of the subintervals 1) 18:07 UT-18:12 UT 2) 18:12 UT-18:27 UT and 3) 18:27 UT-19.03 UT. In Fig. 6 we show the superposition of these results. The MICA curve was added to the figure. As there is a gap of $10 \mathrm{~min}$ of images between the TRACE first interval and MICA's one, in order to interpolate the TRACE curve we added the MICA bifurcation point to the TRACE data. The fact that the TRACE interpolation was slightly shifted in time could be due to differences in the telescope imaging or to the fact that TRACE data are unbalanced with respect to MICA's ones due to the 10 min gap. Figure 6 shows the common pattern of the three time sub-intervals. 


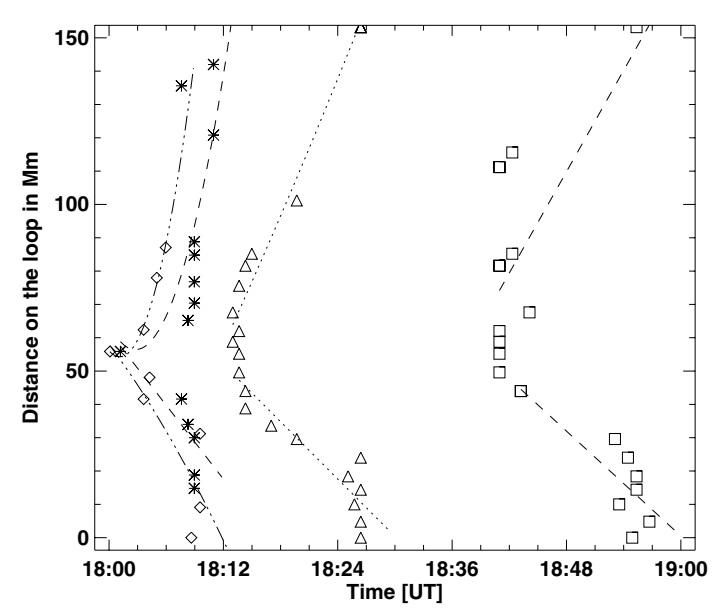

Fig. 6. Superposition of the different events: locations of the brightenings as a function of time measured along the Structure I loop in TRACE images: $*$ for the first TRACE sub-interval; $\triangle$ for the second TRACE sub-interval and $\square$ for the third TRACE sub-interval. To compare the two telescope results the MICA curve of Paper I was superposed $(\diamond)$ on the TRACE first case. In the figure the apex of the Structure is located at $31 \mathrm{Mm}$.

We can distinguish the bifurcation position from where we detect the decrease of the brightenings on both branches. The bifurcation point is located at $d_{1}=56 \pm 5 \mathrm{Mm}$ for the first interval. The decrease seems to occur in approximately $12 \mathrm{~min}$ (as registered by both telescopes) and to occur two times (in approximately 13-18 $\mathrm{min}$ ) from the first appearance of the phenomenon (as described by TRACE). The bifurcation point is more broadly defined in the other intervals: at $d_{2}=55 \pm 10 \mathrm{Mm}$ and $d_{3}=65 \pm 15 \mathrm{Mm}$ respectively.

From the resulting interpolated curves we estimated the decrease velocities of the brightenings of the different curves. The values for the upper parts of Fig. 6 (i.e., the southern branch of the loop) are 1) $v_{\mathrm{S} 1}=150 \pm 25 \mathrm{~km} \mathrm{~s}^{-1}$ (MICA's estimated value was $v_{\mathrm{S} 1}=140 \mathrm{~km} \mathrm{~s}^{-1}$ ) 2) $v_{\mathrm{S} 2}=127 \pm 20 \mathrm{~km} \mathrm{~s}^{-1}$ 3) $v_{\mathrm{S} 3}=85 \pm$ $30 \mathrm{~km} \mathrm{~s}^{-1}$ and, for the lower parts of the same figure (i.e., the northern branch of the loop): 1) $v_{\mathrm{N} 1}=41 \pm 20 \mathrm{~km} \mathrm{~s}^{-1}$ (MICA's estimated value was $v_{\mathrm{N} 1}=41 \mathrm{~km} \mathrm{~s}^{-1}$ ) 2) $v_{\mathrm{N} 2}=71 \pm 25 \mathrm{~km} \mathrm{~s}^{-1}$ 3) $v_{\mathrm{N} 3}=43 \pm 25 \mathrm{~km} \mathrm{~s}^{-1}$. The error estimations were performed following the steps described in Paper I. Note that there is no observational evidence of the reverse effect, this is, we have not detected the ascension of brightenings along the loops. We also could determine that the intensity amplitude decreases towards the footpoints as approximately $10 \%$ to $15 \%$.

To analyze the recurrence and extent of the phenomenon, we applied the procedure to Structure IV. Figure 7 displays, for each cut, the intensity profiles as a function of time. In this Structure, the major activity occurred between 17:06 UT and 17:30 UT. Two other sets of common features were observed to occur around 18:30 UT and around 19:00 UT. These two events occurred at almost the same time as the second and third event of Structure I. Also, the first major activity around 17:15 UT of Structure IV can be correlated with a minor activity detected in the Structure I pattern as seen from the comparison between Figs. 7 and 4a.

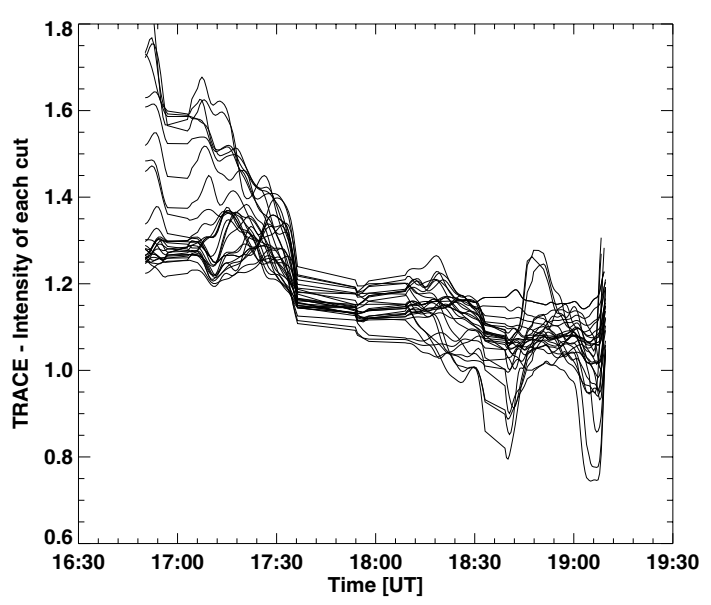

Fig. 7. TRACE Structure IV intensity of each cut as a function of time.

Thus, following the steps performed on Structure I we divided the whole interval in sub-intervals in order to isolate common pattern activities. The sub-intervals chosen were: 1) 17:06 UT-17:30 UT; 2) 17:30 UT-18:36 UT; 3) 18:36 UT-19:06 UT. Then, we applied the method to each sub-interval. Figure $8 \mathrm{a}-\mathrm{c}$ shows, for each of the time subintervals, the tracking of the brightenings along the compound of threads of Structure IV. As was mention Fig. 8 displays the locations where the maxima occur. Again, we found that each of the brightenings have a bifurcation point but in this case we found a different behavior depending on the particular sub-interval i.e. the first sub-interval showed a descending pattern while the other two an ascending one. In these last two cases we found that the intensity amplitude diminished almost by $10 \%$ towards the apex while in the first case (as in Structure I) the amplitude increased almost up to $10 \%$ towards the apex. The cadence of each event was approximately $18 \mathrm{~min}$, $6 \mathrm{~min}$ and $18 \mathrm{~min}$ respectively.

\section{Discussion and conclusions}

In order to gain insight into the physical interpretation of loop dynamics we have implemented a diagnostic method describing intensity variations, both in space and time, along coarse grain loop structures. Also, as was shown in Paper I, the method allows the discrimination between the motion of the whole magnetic structure and the motion of the internal plasma. The method can be applied to any sequence of images obtained with instruments capable of providing images of off-limb loop-like structures with high temporal resolution.

To compare data with the results obtained with the MICA land-based telescope we applied the method to TRACE spaceborn images of loop structures that had the same time and space location as MICA's ones. We could describe a situation (Structure I) where the emission initially developed near the loop top and later slides down on both sides of the loop. The sliding down seemed to occur over approximately $12 \mathrm{~min}$ (as registered by both telescopes) but two reiterations of the phenomenon was then recorded by TRACE (occurring in approximately 13 and $18 \mathrm{~min}$ respectively). Table 1 summarizes the results of Structure I. A different behavior was found when 

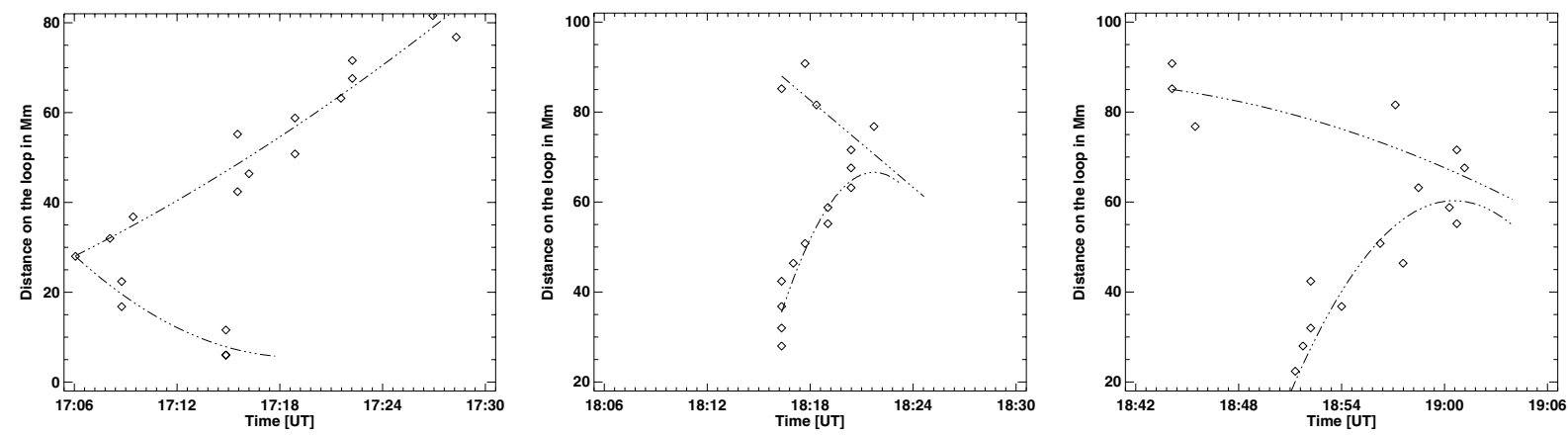

Fig. 8. Structure 4: location of the brightening as a function of time measured along the Structure IV loop in TRACE images for a) the first; b) the second and c) the third sub-intervals respectively. The apex is located at $42 \mathrm{Mm}$ (it was measured along the loop from the first cut).

Table 1. Summary of TRACE Structure I results.

\begin{tabular}{ccccc}
\hline \hline Interval (UT) & Bifurcation point $(\mathrm{Mm})$ & Southern branch speed $\mathrm{km} \mathrm{s}^{-1}$ & Northern branch speed $\mathrm{km} \mathrm{s}^{-1}$ & Time duration of the event \\
\hline $18: 00-18: 12$ & $56 \pm 5$ & $150 \pm 25$ & $41 \pm 20$ & 12 min \\
$18: 12-18: 27$ & $55 \pm 10$ & $127 \pm 20$ & $71 \pm 25$ & 13 min \\
$18: 27-19: 03$ & $65 \pm 15$ & $85 \pm 30$ & $43 \pm 25$ & 18 min \\
\hline
\end{tabular}

Table 2. Summary of TRACE Structure IV results.

\begin{tabular}{ccccc}
\hline \hline Interval (UT) & Bifurcation point $(\mathrm{Mm})$ & Southern branch speed km s & Northern branch speed $\mathrm{km} \mathrm{s}^{-1}$ & Time duration of the event \\
\hline $17: 06-17: 30$ & $27 \pm 7$ & $38 \pm 15$ & $35 \pm 10$ & 18 min \\
$17: 30-18: 36$ & $65 \pm 10$ & $50 \pm 15$ & $156 \pm 20$ & 6 min \\
$18: 36-19: 06$ & $60 \pm 12$ & $17 \pm 20$ & $57 \pm 20$ & 18 min \\
\hline
\end{tabular}

analyzing Structure IV. The first event of this Structure resulted, as in Structure I, of the "sliding down" type, with amplitudes growing toward the loop top. However, in the second and third event we found that the propagation was in the opposite direction; the emission started at the loop basin and developed towards the top together with a smooth decaying of the amplitudes. Table 2 summarizes the results of Structure IV.

The differences in the results obtained with land and space telescopes can be attributed to the fact that variations in the intensity are produced by disturbances that are formed close to the peak formation temperature of the instruments bandpass and thus, as different bandpasses have different temperatures, the disturbances observed are also different. Besides, different spectral components have different dissipation lengths and the wave spectrum evolves with distance along the loop. Also, each of the telescopes record different speeds and thus they describe a different dynamic picture. The temperatures associated with the $17.1 \mathrm{~nm}$ TRACE bandpass is $1.0 \mathrm{MK}$ and the temperature associated with the $530 \mathrm{~nm}$ MICA bandpass is $1.8 \mathrm{MK}$ with corresponding sound speeds of $152 \mathrm{~km} \mathrm{~s}^{-1}$ and $223 \mathrm{~km} \mathrm{~s}^{-1}$ respectively.

As was mentioned, intensity variations in multi-loop systems can be attributed to several-mechanisms among which the discrimination between waves or flow is one of the most important differences. Some of our results that suggest wave-based model interpretations are (DeForest \& Gurman 1998): 1) the periodic behavior of the disturbances; 2) the almost constant speed features of some of the branches of Structure I and IV; and 3) the fact that the estimated speeds of each of the branches are not higher than the sound speed in the coronal loops. However, none of these arguments are conclusive. The periodic behavior can also be attributed to flows (Gómez et al. 1990; De Groof et al. 2004). Also, even when the calculated speeds are not greater than the sound one, from Figs. 6 and 8 it can be noted that some of the velocity patterns are far from being constant and can be compared with the free-fall case. The corresponding free fall times of each of the time intervals of Structure I are: 10:30 min, $11 \mathrm{~min}$ and 11:25 min respectively and for Structure IV: 7:20 $\mathrm{min}, 10 \mathrm{~min}$ and $9 \mathrm{~min}$ respectively. Figure 9 compares the second interval of Structure IV with the reverse of a free-fall speed diagram, both with the same initial vertical upward speed. This interval is better described by the free-fall speed diagram than the other ones. Moreover, most of the branch velocities are significantly below the expected sound speed and, in addition, the speeds of brightening variations obtained from MICA images are not higher than the TRACE ones as is expected from the higher temperature disturbances recorded by MICA.

We found that in every case where the descending event was registered, the amplitude of the perturbation was larger $( \pm 10 \%)$ at the apex than at the footpoints. This is consistent with observations in solar plumes that were interpreted as the propagation of slow magnetosonic waves from the bases that are nonlinearly steepened with height by generating higher 


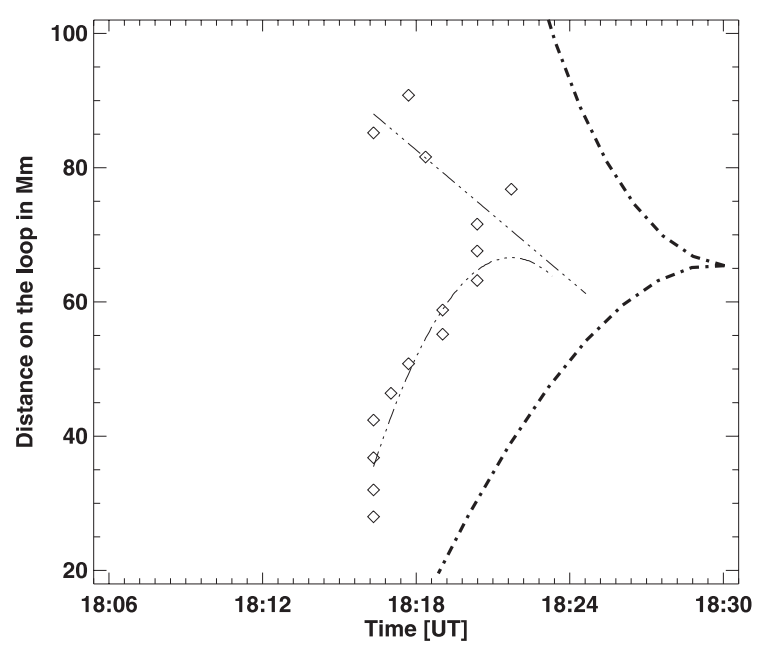

Fig. 9. Structure 4: comparison of the second interval with the free-fall case.

harmonics leading to enhanced dissipation (Ofman et al. 1999; Tsiklauri \& Nakariakov 2001). In the framework of investigating the feasibility of a wave interpretation a speculative hypothesis to explain why only the sliding down was detected is related with the fact that, on the contrary with common observations, the intensity maxima or the amplitude saturation of the nonlinear steepening and trapping of the waves we observed occur at the apex of the loops, i.e., at the end of the propagating process and not at the beginning of the wave propagation from the bases. This could led to the conclusion that the registered event is the tracking with time of the intensity perturbation from the top where the maximum intensity occurs towards the bases where the decay is significant. In this context, the fact that there are no observations of downward propagation disturbances in coronal plumes could be ascribed to the particular feature of diverging magnetic field lines that characterizes these structures in comparison with loop-like ones. A more usual wave interpretation could be argued to explain Structure IV: ascending features with decaying intensities towards the apex suggest that descending amplitudes must be much weaker and efficiently depressed by dissipation processes and thus, they are pooely observed (Ofman et al. 1999; Nakariakov et al. 2000; King et al. 2003).

On the other hand, in the framework of flow interpretations, our results are in accordance with a catastrophic evacuation or high-speed descending flow picture sustained by several authors e.g. Kjeldseth-Moe \& Brekke (1998), Schrijver (2001). Another explanation suggests that small-scale flaring in coronal loops produces a descending appearance attributed to the draining of the material (Harra et al. 2004). This characterization is difficult to sustain in this context as it requires an explanation for the upward behavior pattern and the quasi-periodic iteration of the events. However, limit cycle solutions of a compound of coherent non-resolved loops that assume an evaporationcondensation mechanism seem more promising for interpreting our observations (e.g., Gómez et al. 1990; De Groof et al. 2004).
In any case, as was mentioned in the introduction, due to the flaring character of the event a combination of the two descriptions (flow and waves) could give a better interpretation. The series of simulations (Tsiklauri et al. 2004a,b,c) suggesting that up-flow evaporation and posterior draining of material from post-flare loops (with speeds of hundreds of $\mathrm{km} \mathrm{s}^{-1}$ ) could occur together with the appearance of oscillatory patterns in the physical quantities (with speeds of about few tens of $\mathrm{km} \mathrm{s}^{-1}$ ) seem closer to observations. Due to the simplifications that are assumed when simulating physical quantities (e.g. a peak of heat deposition at the bottom of the loop in a short time interval) the theoretical descriptions generally allow us to distinguish the behavior of the different components: flow and waves. But, in observational scenarios the dynamic is generally much more complex, making it difficult to isolate the contribution of each component e.g., the erratic flaring from the oscillations. Thus, in the framework of this reasoning, we can argue that the broad spectrum of different speeds (with differences in magnitude: from a few tens to hundreds of $\mathrm{km} \mathrm{s}^{-1}$ and variations along the loop: almost constant to comparable with a free-fall description) that we have obtained could be due to the combined action of both type of phenomena.

The different apparently isolated filaments evolve in a coherent way. All the threads in the region covered by the cuts exhibit their maxima of activity in the same time sub-intervals, resembling either the coherent sliding down of flow (or perturbations) from the top of the structure or the upward behavior of flow (or perturbations) from the base. The fact that there are various data points with the same time position and different distances along the loop (necessarily corresponding to different threads) is also a consequence of the coherence in the behavior of the region. This is in accordance with the description of an individual non-resolved loop-like structure described in Paper I and could be the explanation of the fact that we detected two maxima at the same time (see Fig. 5a).

Also, from a global comparison between the two Structures, Figs. 4a and 7, we can see that there is a time correlation between relative maxima. The two structures have maxima in the time intervals: $17: 15-17: 25,18: 15-18: 20$ and 18:50-18:55. Thus, the apparent coronal isolation of the magnetic threads suggest that the coherence in the behavior of the whole event is due to similar conditions at the base or at the top of the two structures. In fact, the reports by Schrijver (2001), Harra et al. 2004 and Nakariakov et al. (2000) suggest that the triggering mechanism that gives rise to similar conditions must be located at the top of the structures, while the papers by De Groof et al. (2004), Tsiklauri et al. (2004a,b,c) and Gómez et al. (1990) suggest that the mechanism is located at the chromospheric bottom of the structures. However, it seems most possible that the intermittent flaring of the whole region is responsible for the coherence between both Structures. Taking into account the time correlation between the main patterns of Structures I and IV we could determine a $360 \mathrm{Mm}$ longitude of coherence for the whole phenomenon.

Acknowledgements. We are grateful to Dr. Estela Reynoso for her comments and suggestions that helped to improve the quality of the paper. 


\section{References}

Alexander, D., Harra-Murnion, L. K., Khan, J. I., \& Matthews, S. A. 1998, ApJ, 494, L235

Aschwanden, M. J., Newmark, J. S., Delaboudiniere, J.-P., et al. 1999, ApJ, 515, 842

Aschwanden, M. J., Alexander, D., Hurlburt, N., et al. 2000, ApJ, 531, 1129

Costa, A., \& Stenborg, G. 2004, Sol. Phys., 222, 2

Craig, I. J., \& McClymont, A. 1978, A\&A, 70, 1

De Groof, A., Berghmans, D., van Driel-Gesztelyi, L., \& Poedts, S. 2004, A\&A, 415, 1141

Delaboudiniere, J.-P., Artzner, G. E., Brunaud, J., et al. 1995, Sol. Phys., 162, 291

DeForest, C. E., \& Gurman, J. B. 1998, ApJ, 501, L217

Gómez, D., Sicardi Schifino, A., \& Ferro Fontán, C. 1990, ApJ, 352, 318

Handy, B. N., Acton, L. W., \& Kankelborg, C. C. 1999, Sol. Phys., 184,229

Harra, L. K., Mandrini, C. H., \& Matthews, S. A. 2004, Sol. Phys., 223, 57

Jordan, C. 1980, A\&A, 86, 355

King, D. B., Nakariakov, V. M., Deluca, E. E., Golub, L., \& McClements, K. G. 2003, A\&A, 404, L1

Kjeldseth-Moe, O., \& Brekke, P. 1998, Sol. Phy., 182, 73
Klimchuk, J. A., \& Mariska, J. T. 1988, ApJ, 328, 324

Mariska, J. T. 1984, ApJ, 281, 435

Nakariakov, V. M., Verwichte, E., Berghmans, D., \& Robbrecht, E. 2000, A\&A, 362, 1151

Nakariakov, V. M., Tsiklauri, D., Kelly, A., Arber, T. D., \& Aschwanden, M. J. 2004, A\&A, 414, L25

Ofman, L., Nakariakov, V. M., \& DeForest, C. E. 1999, ApJ, 514, 441

Schrijver, C. J. 2001, Sol. Phys., 198, 325

Serio, S., Peres, G., Vaiana, G. S., Golub, L., \& Rosner, R. 1981, ApJ, 243, 288

Stenborg, G., Schwenn, R., Srivastava, N., et al. 1999, Space Sci. Rev., 87,307

Tsiklauri, D., \& Nakariakov, V. M. 2001, A\&A, 379, 1106

Tsiklauri, D., Aschwnaden, M. J., Nakariakov, V. M., \& Arber, T. D. 2004a, A\&A, 419, 1149

Tsiklauri, D., Nakariakov, V. M., Arber, T. D., \& Aschwnaden, M. J. 2004b, A\&A, 422, 351

Tsiklauri, D., Nakariakov, V. M., Arber, T. D., \& Aschwnaden, M. J. 2004c, Proc. of the SOHO 15 Workshop - Coronal Heating. 6-9 September 2004, St. Andrews, Scotland, UK (ESA SP575), ed. R. W. Walsh, J. Ireland, D. Danesy, \& B. Fleck, Paris: European Space Agency

Walsh, R. W., \& Galtier, S. 2000, Sol. Phys., 197, 57

Wood, J. W., Karakovska, M., Cook, J. W., et al. 1998, ApJ, 505, 432 